\title{
THE LOW-FREQUENCY CHARACTERISTICS OF PSR J0437-4715 OBSERVED WITH THE MURCHISON WIDE-FIELD ARRAY
}

\author{
N. D. R. Bhat ${ }^{1,2}$, S. M. Ord ${ }^{1,2}$, S. E. Tremblay ${ }^{1,2}$, S. J. Tingay ${ }^{1,2}$, A. A. Deshrande ${ }^{3}$, W. van Straten $^{2,4}$, S. Oronsaye ${ }^{1,2}$, \\ G. Bernardi ${ }^{5,6,7}$, J. D. Bowman ${ }^{8}$, F. Briggs ${ }^{2,9}$, R. J. CAPPAllo ${ }^{10}$, B. E. Corey ${ }^{10}$, D. Emrich ${ }^{1}$, R. Goeke ${ }^{11}$, L. J. Greenhill ${ }^{7}$, \\ B. J. Hazelton ${ }^{12}$, J. N. HewitT ${ }^{11}$, M. Johnston-Hollitt ${ }^{13}$, D. L. Kaplan ${ }^{14}$, J. C. Kasper ${ }^{7,15}$, E. KratzenberG ${ }^{10}$, \\ C. J. Lonsdale ${ }^{10}$, M. J. LynCh $^{1}$, S. R. McWhirter ${ }^{10}$, D. A. Mitchell ${ }^{2,16}$, M. F. Morales ${ }^{12}$, E. Morgan ${ }^{11}$, D. Oberoi ${ }^{17}$, \\ T. Prabu ${ }^{3}$, A. E. E. Rogers ${ }^{10}$, D. A. Roshi ${ }^{18}$, N. Udaya Shankar ${ }^{3}$, K. S. Srivani ${ }^{3}$, R. Subrahmanyan ${ }^{2,3}$, M. WAterson $^{1,9}$, \\ R. B. Wayth ${ }^{1,2}$, R. L. Webster ${ }^{2,19}$, A. R. Whitney ${ }^{10}$, A. Williams ${ }^{1}$, and C. L. Williams ${ }^{11}$ \\ ${ }^{1}$ International Centre for Radio Astronomy Research, Curtin University, Bentley, WA 6102, Australia \\ ${ }^{2}$ ARC Centre of Excellence for All-sky Astrophysics (CAASTRO), Curtin University, Bentley, WA 6102, Australia \\ ${ }^{3}$ Raman Research Institute, Bangalore 560080, India \\ ${ }^{4}$ Centre for Astrophysics and Supercomputing, Swinburne University, Hawthorn, Victoria 3122, Australia \\ ${ }^{5}$ Square Kilometre Array South Africa, 3rd Floor, The Park, Park Road, Pinelands, 7405, South Africa \\ ${ }^{6}$ Department of Physics and Electronics, Rhodes University, P.O. Box 94, Grahamstown, 6140, South Africa \\ ${ }^{7}$ Harvard-Smithsonian Center for Astrophysics, Cambridge, MA 02138, USA \\ ${ }^{8}$ School of Earth and Space Exploration, Arizona State University, Tempe, AZ 85287, USA \\ ${ }^{9}$ Research School of Astronomy and Astrophysics, Australian National University, Canberra, ACT 2611, Australia \\ ${ }^{10}$ MIT Haystack Observatory, Westford, MA 01886, USA \\ ${ }^{11}$ Kavli Institute for Astrophysics and Space Research, Massachusetts Institute of Technology, Cambridge, MA 02139, USA \\ ${ }^{12}$ Department of Physics, University of Washington, Seattle, WA 98195, USA \\ ${ }^{13}$ School of Chemical \& Physical Sciences, Victoria University of Wellington, Wellington 6140, New Zealand \\ ${ }^{14}$ Department of Physics, University of Wisconsin-Milwaukee, Milwaukee, WI 53201, USA \\ ${ }^{15}$ Department of Atmospheric, Oceanic and Space Sciences, University of Michigan, Ann Arbor, MI 48109, USA \\ ${ }^{16}$ CSIRO Astronomy and Space Science, Marsfield, NSW 2122, Australia \\ ${ }^{17}$ National Centre for Radio Astrophysics, Tata Institute for Fundamental Research, Pune 411007, India \\ ${ }_{18}^{18}$ National Radio Astronomy Observatory, Charlottesville and Greenbank, USA \\ ${ }^{19}$ School of Physics, The University of Melbourne, Parkville, VIC 3010, Australia \\ Received 2014 May 23; accepted 2014 July 16; published 2014 August 6
}

\begin{abstract}
We report on the detection of the millisecond pulsar PSR J0437-4715 with the Murchison Wide-field Array (MWA) at a frequency of $192 \mathrm{MHz}$. Our observations show rapid modulations of pulse intensity in time and frequency that arise from diffractive scintillation effects in the interstellar medium (ISM), as well as prominent drifts of intensity maxima in the time-frequency plane that arise from refractive effects. Our analysis suggests that the scattering screen is located at a distance of $\sim 80-120$ pc from the Sun, in disagreement with a recent claim that the screen is closer $(\sim 10 \mathrm{pc})$. Comparisons with higher frequency data from Parkes reveal a dramatic evolution of the pulse profile with frequency, with the outer conal emission becoming comparable in strength to that from the core and inner conal regions. As well as demonstrating the high time resolution science capabilities currently possible with the MWA, our observations underscore the potential to conduct low-frequency investigations of timing-array millisecond pulsars, which may lead to increased sensitivity in the detection of nanoHertz gravitational waves via the accurate characterization of ISM effects.
\end{abstract}

Key words: instrumentation: interferometers - methods: observational - pulsars: general pulsars: individual (PSR J0437-4715)

Online-only material: color figures

\section{INTRODUCTION}

PSR J0437-4715, the closest and brightest millisecond pulsar (MSP), has been intensely studied ever since its discovery (Johnston et al. 1993). This $5.75 \mathrm{~ms}$ pulsar in a nearly circular 5.74 day orbit with a low-mass $\left(\approx 0.24 M_{\odot}\right)$ helium white dwarf companion has also been detected at optical, ultraviolet, X-ray, and $\gamma$-ray wavelengths (Bell et al. 1993; Kargaltsev et al. 2004; Zavlin et al. 2002; Abdo et al. 2013). It has the lowest dispersion measure (DM) of all known MSPs $\left(2.65 \mathrm{pc} \mathrm{cm}^{-3}\right)$ and is located at a distance of $157 \pm 3$ pc (Verbiest et al. 2008; Deller et al. 2008). PSR J0437-4715 is among the most important objects for long-term high-precision timing applications and holds the record for timing precision over a decadal time span (rms timing residual $=199 \mathrm{~ns}$; Verbiest et al. 2008). Consequently, it is among the most promising objects for pulsar timing-array experiments that aim to detect nanoHertz gravitational waves via high-precision timing. It is a prime target for the ongoing Parkes pulsar timing array (PPTA) project (Manchester et al. 2013), and later this decade, the South African MeerKAT may also be used for its timing observations.

Its proximity makes PSR J0437-4715 an important object for probing the local interstellar medium (ISM), which is known to harbor large-scale features such as the Local Bubble (Snowden et al. 1990; Bhat et al. 1998; Cordes \& Lazio 2002; Spangler 2009). Given its low DM and a location well below the Galactic plane $\left(b \approx-42^{\circ}\right)$, it is likely to be relatively weakly scattered, and therefore observations at the Murchison Wide-field Array (MWA) frequencies $(\lesssim 300 \mathrm{MHz})$ are most ideal to characterize the ISM along its line of sight. However, the reported scintillation work in the published literature is limited, and was undertaken at higher frequencies (Johnston 
et al. 1998; Gothoskar \& Gupta 2000; Gwinn et al. 2006). Even though scattering and refraction are generally expected to be negligible at frequencies $\gtrsim 1 \mathrm{GHz}$, where high-precision timing is currently achievable, the PPTA data show significant $\mathrm{DM}$ variations, $\sim 10^{-3} \mathrm{pc} \mathrm{cm}^{-3}$ over time spans of $\sim 5-10 \mathrm{yr}$ (Keith et al. 2013), corresponding to timing perturbations of $\sim 1-2 \mu \mathrm{s}$. The dispersive delays within the MWA band will be 1-2 orders of magnitude larger than those at $\sim 700 \mathrm{MHz}$, the lowest frequency of PPTA observations. Low-frequency investigations are therefore particularly important in order to characterize the ISM effects toward this pulsar.

PSR J0437-4715 also has a remarkable pulse profile, comprising multiple overlapping components, with emission extending out to nearly $85 \%$ of the rotation period (Johnston et al. 1993; Manchester \& Johnston 1995; Yan et al. 2011). It is also very well studied in polarization (e.g., Manchester \& Johnston 1995; Navarro et al. 1997), and is an important polarimetric calibrator for timing-array experiments and high-precision pulsar polarimetry (e.g., van Straten 2013).

While the PPTA observations are made at frequencies $\gtrsim 700 \mathrm{MHz}$ (Manchester et al. 2013), the pulsar has previously been detected at lower frequencies, down to $76 \mathrm{MHz}$ (McConnell et al. 1996), and also at 151 and $330 \mathrm{MHz}$ (Issur \& Deshpande 2000; Vivekanand et al. 1998), though the latter two used instruments of single polarization. Despite their limited time resolution and signal-to-noise ratio, a strong frequency evolution of the pulse profile was hinted at by these early observations.

In this Letter, we report on the detection of pulsar PSR J0437-4715 made with the MWA. Our observations enable the lowest-frequency scintillation studies ever made of this pulsar, and reveal the signatures of both diffractive and refractive scintillation effects. Details of our observations and data processing are summarized in Section 2, a discussion of the results is in Section 3, and future prospects are outlined in Section 4.

\section{OBSERVATIONS AND DATA PROCESSING}

The MWA is a newly operational low-frequency array that comprises 128 tiles operating from 80 to $300 \mathrm{MHz}$ (Tingay et al. 2013). While originally conceived primarily as an imaging instrument (Lonsdale et al. 2009), the array is equipped with a high time resolution data recorder, the voltage capture system (VCS), to enable time-domain science applications such as observations of pulsars and fast radio bursts. Implementation details of the VCS will be described elsewhere (S. E. Tremblay et al. in preparation). This functionality allows recording up to $24 \times 1.28 \mathrm{MHz}$ from all 128 tiles (both polarizations). These 24 coarse channels are further subdivided into $10 \mathrm{kHz}$ fine channels, resulting in a native time resolution of $100 \mu \mathrm{s}$. Recording at $12 \times 1.28 \mathrm{MHz}$ is currently possible, providing a net bandwidth of $15.36 \mathrm{MHz}$, and the full-bandwidth capability is anticipated later in 2014. Commissioning observations to date have resulted in the detection of eight pulsars, including the Crab and PSR J0437-4715.

Observations of PSR J0437-4715 were made on two separate occasions, on 2013 September 25 and 2013 December 13, and the data were recorded over a $12 \times 1.28 \mathrm{MHz}$ bandwidth centred at $192.6 \mathrm{MHz}$. For each observation, the raw voltage data from VCS, at a rate of $328 \mathrm{MB} \mathrm{s}^{-1}$ per $1.28 \mathrm{MHz}$ coarse channel (i.e., four-bit sampling), were recorded onto disks for a duration of $1 \mathrm{hr}$, resulting in an aggregate $13.5 \mathrm{~TB}$ of data per observation. Owing to a temporary limitation with our data recording, only 88 of the $128 \times 10 \mathrm{kHz}$ channels (in each coarse channel) were
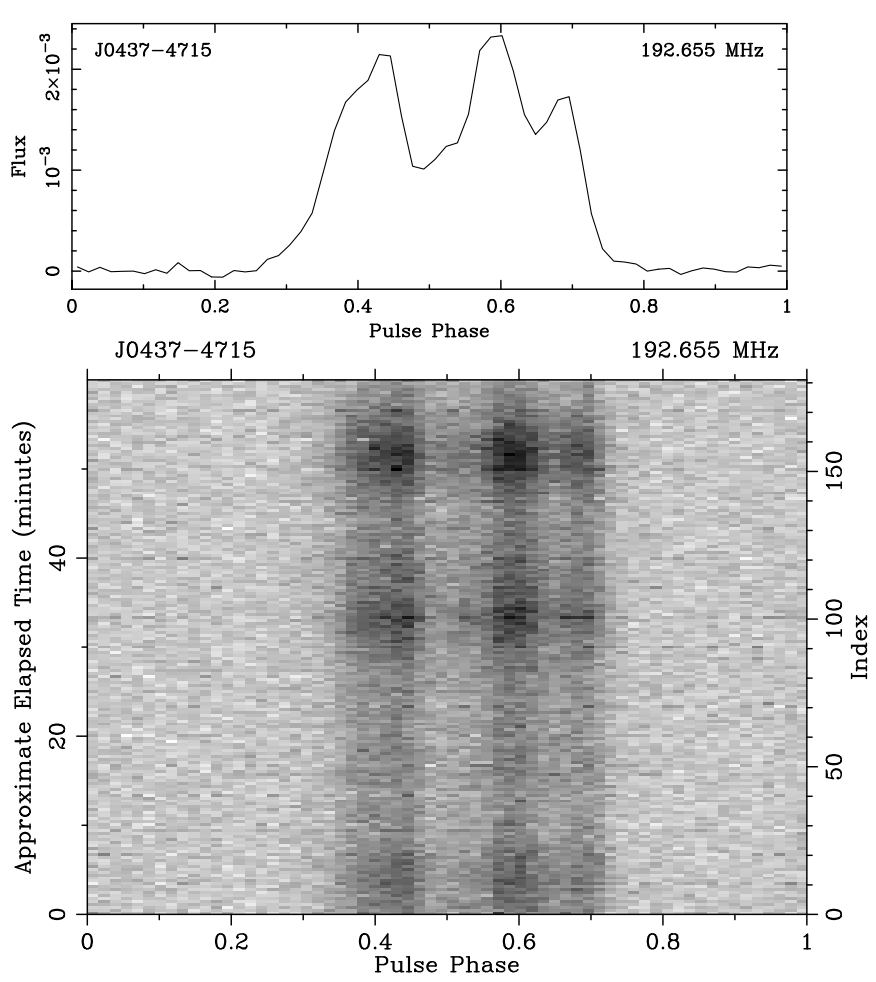

Figure 1. Detection of PSR J0437-4715 with the MWA. The integrated pulse profile (top) and the pulse strength vs. time and pulse phase (bottom) are shown for the $1 \mathrm{hr}$ of observation centered at $192.6 \mathrm{MHz}$ over a bandwidth of $15.36 \mathrm{MHz}$ ("index" on right-hand side of the bottom panel refers to a $20 \mathrm{~s}$ sub-integration). Data are de-dispersed, and the residual dispersive smearing within $10 \mathrm{kHz}$ channels is $\approx 35 \mu \mathrm{s}$, smaller than the $100 \mu \mathrm{s}$ time resolution.

written to the disks. These data were then processed to form an incoherent addition of detected powers from all 128 tiles, and were written out in the PSRFITS data format (Hotan et al. 2004) after summing the two linear polarizations. The resultant spectra have a temporal resolution of $100 \mu$ s and a spectral resolution of $10 \mathrm{kHz}$.

Figure 1 shows the pulsar detection from our first observation. With a signal-to-noise ratio of $\mathrm{S} / \mathrm{N} \sim 205$, the implied mean pulsar flux is $\sim 2 \mathrm{Jy}$, assuming nominal MWA sensitivity. In the second observation, the pulsar was significantly weaker $(\mathrm{S} / \mathrm{N} \sim$ 80 ), even though the array had $\sim 85 \%$ of its nominal sensitivity; the change in flux (down to $\sim 1 \mathrm{Jy}$ ) is thus primarily due to scintillation.

The use of the PSRFITS data format provides compatibility with standard pulsar software packages, PRESTO (Ransom 2001) and DSPSR (van Straten \& Bailes 2011). Initial processing and checks were performed using PRESTO, following which the data were processed using DSPSR to generate synchronously folded pulse profiles over $20 \mathrm{~s}$ sub-integrations. These were subsequently averaged in frequency for the improved signal-tonoise ratio needed to generate a dynamic spectrum, shown in Figure 2. A resolution of $0.64 \mathrm{MHz}$ was chosen to minimize instrumental artifacts that may arise from periodic flagging of the edge channels (20 each) on either end of a given coarse (1.28 MHz) channel.

\section{RESULTS AND DISCUSSION}

Our data show rapid, deep modulations of pulse intensity in time and frequency arising from diffractive scintillation, as well as prominent drifts of scintillation maxima arising from refractive effects caused by discrete, wedge-like density structures. 


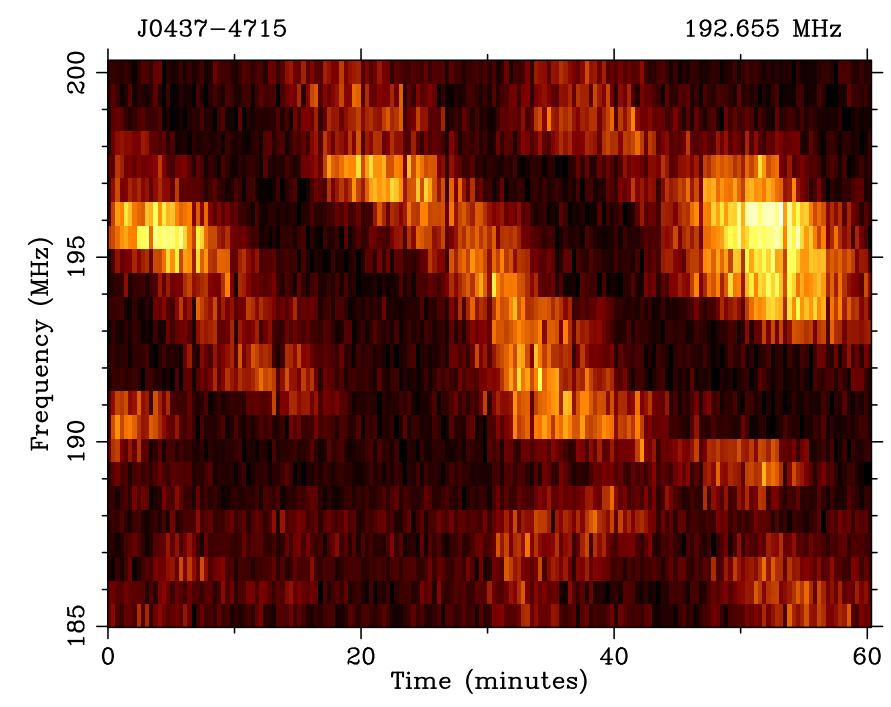

Figure 2. Dynamic spectrum of the pulse intensity from the MWA observations of PSR J0437-4715 over a time duration of $1 \mathrm{hr}$ and a bandwidth of $15.36 \mathrm{MHz}$ centered at $192.655 \mathrm{MHz}$. The time and frequency resolutions in this plot are $20 \mathrm{~s}$ (i.e., one sub-integration) and $0.64 \mathrm{MHz}$, respectively.

(A color version of this figure is available in the online journal.)

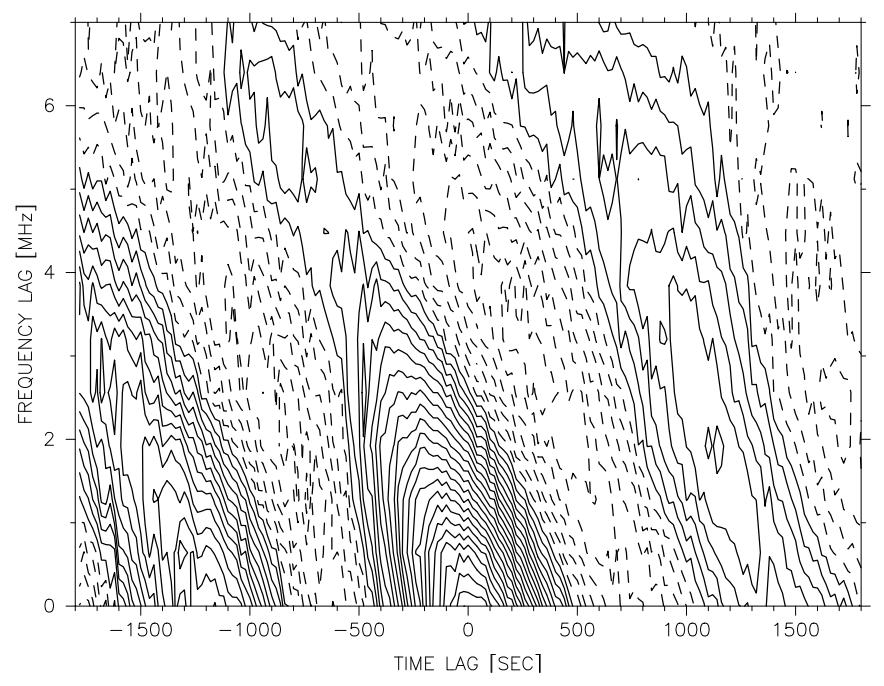

Figure 3. Auto-correlation function of the dynamic spectrum of PSR J0437-4715 shown in Figure 2, plotted for lag values out to half the total observing time and half the observing bandwidth.

Although scintillation observations of PSR J0437-4715 have been made in the past (Nicastro \& Johnston 1995; Johnston et al. 1998; Gothoskar \& Gupta 2000; Gwinn et al. 2006), this is the first time such drifts have been observed for this pulsar. Characteristic scales in time and frequency, and the drift rate in the time-frequency plane, can be derived by computing the autocorrelation function (ACF) of the dynamic spectra (Figure 3). The secondary maxima in this plot are due to a few dominant scintles being present in our data (Figure 2).

Following the published literature (Gupta et al. 1994; Bhat et al. 1999; Wang et al. 2005), we fit the ACF with a two-dimensional elliptical Gaussian of the form $\rho_{g}(\nu, \tau)=$ $C_{0} \exp \left[-\left(C_{1} \nu^{2}+C_{2} v \tau+C_{3} \tau^{2}\right)\right]$ to yield characteristic scales: the decorrelation bandwidth, $v_{d}=\left(\ln 2 / C_{1}\right)^{0.5}$, measured as the half-width at half-maximum of the correlation peak; the scintillation timescale, $\tau_{\text {iss }}=\left(1 / C_{3}\right)^{0.5}$, measured as the $1 / e$ width of the correlation function; and the drift rate, $d t / d v=$
Table 1

Scintillation Measurements of PSR J0437-4715

\begin{tabular}{lccccccc}
\hline \hline MJD & $\begin{array}{c}v_{\text {obs }} \\
(\mathrm{MHz})\end{array}$ & $\begin{array}{c}v_{\mathrm{d}} \\
(\mathrm{MHz})\end{array}$ & $\begin{array}{c}v_{\mathrm{d}, \mathrm{mwa}} \mathrm{b} \\
(\mathrm{MHz})\end{array}$ & $\begin{array}{c}\tau_{\text {iss }} \\
(\mathrm{min})\end{array}$ & $\begin{array}{c}\tau_{\text {iss,mwa }} \mathrm{b} \\
(\mathrm{min})\end{array}$ & $\begin{array}{c}V_{\text {iss }} \\
\left(\mathrm{km} \mathrm{s}^{-1}\right)\end{array}$ & Ref. \\
\hline 48825 & 436 & $3.2-4.4$ & $0.13-0.18$ & $4.6-10.9$ & $1.7-4.1$ & $114-288^{\mathrm{a}}$ & 1 \\
49459 & 436 & 3.6 & 0.15 & 7.8 & 2.9 & 170 & 2 \\
& 660 & 17.4 & 0.14 & 10 & 2.3 & & 2 \\
50523 & 327 & $0.2-3.0$ & $0.03-0.38$ & $1.8-5.0$ & $1.0-2.6$ & $171-321$ & 3 \\
50392 & 328 & 16 & 1.98 & 17 & 9 & $110-200$ & 4 \\
56559 & 328 & 0.5 & 0.06 & 1.5 & 0.8 & & 4 \\
& 192 & 1.7 & 1 & 4.5 & 1 & $325 \pm 90$ & 5 \\
\hline
\end{tabular}

Notes.

a Rescaled for $A_{\text {ISS }}=3.85 \times 10^{4}$ (see Gupta et al. 1994), the adopted value for all other Refs.

b Scaled to the MWA frequency $\nu_{\mathrm{d} \text {,mwa }}=192 \mathrm{MHz}$ using a scaling index, $\alpha=$ 3.9 , for $v_{\mathrm{d}} \propto\left(v_{\mathrm{obs}}\right)^{\alpha}$ (see Bhat et al. 2004) and assuming $\tau_{\mathrm{iss}} \propto\left(v_{\mathrm{obs}}\right)^{1.2}$.

References. (1) Nicastro \& Johnston 1995; (2) Johnston et al. 1998; (3) Gothoskar \& Gupta 2000; (4) Gwinn et al. 2006; (5) this work.

$-\left(C_{2} / 2 C_{3}\right)$. The presence of significant refractive bending results in an underestimation of the decorrelation bandwidth, as the drifting patterns are no longer aligned in time; however, this can be alleviated to a certain extent (see Bhat et al. 1999) by the use of a "drift-corrected" decorrelation bandwidth, $v_{\mathrm{d}_{\mathrm{c}}}$, defined as

$$
v_{\mathrm{d}_{\mathrm{c}}}=(\ln 2)^{0.5}\left(C_{1}-\frac{C_{2}^{2}}{4 C_{3}}\right)^{-0.5} .
$$

The measurement errors on these quantities are largely due to the limited number of scintles in our data, given by $\sigma_{f}^{2} \approx$ $(1 / f)\left(\tau_{\text {iss }} / T_{\text {obs }}\right)\left(v_{d} / B\right)$, where $f$ is the filling factor (nominally assumed to be $0.2-0.5), T_{\text {obs }}$ and $B$ are the net observing time and bandwidth, respectively, and $\sigma_{f}$ is the fractional error. For the data shown in Figure 2 , we estimate $v_{\mathrm{d}_{\mathrm{c}}} \sim 1.7 \mathrm{MHz}, \tau_{\text {iss }} \sim$ $260 \mathrm{~s}, d t / d v \sim 95 \mathrm{~s} \mathrm{MHz}^{-1}$, and $\sigma_{f} \sim 0.2$, with a net measurement error $(\sigma) \sim 25 \%$, accounting for $\sim 15 \%$ error from the model fits.

\subsection{Scintillation Measurements}

\subsubsection{Decorrelation Bandwidth and Scintillation Scales}

A summary of all published scintillation measurements for PSR J0437-4715 is given in Table 1. For meaningful comparisons, we have also tabulated the scaled values of the decorrelation bandwidth and scintillation timescale at the MWA's frequency, $v_{\mathrm{d} \text {,mwa }}$ and $\tau_{\text {iss,mwa }}$, respectively. As all measurements including ours are obtained from a single or a few epochs of observation, they are subject to large uncertainties (by factors as much as $\sim 2-3$ ) from refractive scintillation (e.g., Wang et al. 2005; Bhat et al. 1999; Gupta et al. 1994). Our measured $v_{\mathrm{d}}$ implies a wavenumber spectral coefficient, $\overline{C_{\mathrm{n}}^{2}} \sim 9 \times 10^{-5} \mathrm{~m}^{-20 / 3}$, corresponding to a scattering measure, $\mathrm{SM} \equiv \int C_{\mathrm{n}}^{2}(l) d l \sim 4.5 \times 10^{-6} \mathrm{kpc} \mathrm{m}^{-20 / 3}$. These are the second lowest of all measurements published so far. The lowest values of $\overline{C_{\mathrm{n}}^{2}}$ and SM are reported toward PSR B0950+08 (Phillips \& Clegg 1992), which has a slightly higher DM of $2.96 \mathrm{pc} \mathrm{cm}^{-3}$ and is located at a larger distance $(280 \pm 25 \mathrm{pc})$ compared to PSR J0437-4715.

Table 1 indicates that our measured $v_{\mathrm{d}}$ is highly discrepant with the majority of the published values by over an order of magnitude, but agrees with the larger scale of scintillation from 
Gwinn et al. (2006). Further, most published values are, if at all, closer to the smaller scale in their observation. ${ }^{20}$ Gwinn et al. (2006) interpret their observations in terms of "two scales of scintillation," corresponding to two scales of structure. However, the appearance of two distinct scales (in time and frequency), separated by $\sim 1-2$ orders of magnitude, can occur when two or more scattered sub-images superpose at the observer, giving rise to periodic patterns - “interstellar fringes"-in pulsar dynamic spectra (e.g., Rickett et al. 1997; Gupta et al. 1999). Furthermore, these are expected to be transitory, with variable widths on timescales ranging from days to months (e.g., Rickett et al. 1997), and require more refraction than that predicted by a pure Kolmogorov medium, particularly for observations at frequencies well below the transition frequency $\left(v_{\mathrm{c}}\right)$, where the decorrelation bandwidth $v_{\mathrm{d}} \sim$ the observing frequency, $v_{\mathrm{obs}}$. Our measured $v_{\mathrm{d}}$ implies $v_{\mathrm{c}} \sim 1 \mathrm{GHz}$, and, incidentally, all the reported scintillation measurements of PSR J0437-4715 are from observations at frequencies $\lesssim 600 \mathrm{MHz}$.

\subsubsection{Scintillation Velocity and Location of the Scattering Screen}

Measurements of the decorrelation bandwidth $\left(v_{\mathrm{d}}\right)$ and scintillation timescale $\left(\tau_{\text {iss }}\right)$ can be used to derive the scintillation velocity, $V_{\text {iss }}$, i.e., the net transverse motion between the pulsar, the observer, and the medium. The measured values of $v_{\mathrm{d}}$ and $\tau_{\text {iss }}$ (and hence $V_{\text {iss }}$ ) critically depend on both the line of sight and transverse distributions of scattering material, as well as on the wavenumber spectrum of plasma irregularities. The transverse distribution is particularly important because the physical extent of the screen (or medium) influences the apparent decorrelation bandwidth; for instance, a finite transverse extent of the screen will give rise to a larger value for $v_{d}$ (Cordes \& Lazio 2001). Moreover, statistical inhomogeneities will lead to time-dependent modulations of $v_{\mathrm{d}}$ and $\tau_{\text {iss }}$, as often observed (e.g., Gupta et al. 1994; Bhat et al. 1999); such effects can be more prominent at low frequencies because of inherently wider scattering cones.

Detailed treatments relating the quantities $\nu_{\mathrm{d}}$ and $\tau_{\text {iss }}$ to $V_{\text {iss }}$ are given by Cordes \& Rickett (1998) and Deshpande \& Ramachandran (1998). Their formalisms can be used to derive improved pulsar distance estimates, if the pulsar proper motion $(\mu)$ is also known. In the case of PSR J0437-4715, both the distance and proper motion are very well constrained (Verbiest et al. 2008; Deller et al. 2008), and hence the knowledge of $V_{\text {iss }}$ and proper motion, together with the measurements of $v_{\mathrm{d}}$ and $\tau_{\text {iss }}$, can be used to place constraints on the location of the scattering screen. For the simplest case, where the scattering medium is approximated as a thin screen located between the pulsar and the observer, $V_{\text {iss }}$ is given by (see Gupta et al. 1994)

$$
V_{\mathrm{iss}}=A_{\mathrm{ISS}} \frac{\sqrt{\nu_{\mathrm{d}} D_{\mathrm{psr}} x}}{v_{\mathrm{obs}} \tau_{\mathrm{iss}}},
$$

where $D_{\mathrm{psr}}$ is the pulsar distance, and $x=D_{\mathrm{os}} / D_{\mathrm{ps}}$, i.e., the ratio of the distances from the screen to the observer $\left(D_{\mathrm{os}}\right)$ and from the screen to the pulsar $\left(D_{\mathrm{ps}}\right)$. The constant, $A_{\mathrm{ISS}}$, relates the decorrelation time $\left(\tau_{\text {iss }}\right)$ to the velocity, for which Cordes \& Rickett (1998) derive $2.53 \times 10^{4}$ for a Kolmogorov turbulence spectrum and homogeneously distributed medium, whereas for a single asymmetrically located thin screen, Gupta et al. (1994) derive $A_{\text {ISS }}=3.85 \times 10^{4}$. The scintillation

\footnotetext{
20 Our data do not show any evidence of a smaller scale, though this may be
} due to insufficient signal-to-noise ratio at high $(10 \mathrm{kHz})$ resolution. velocity is essentially a vector addition of the pulsar's transverse velocity, $V_{\mu}=\mu D_{\mathrm{psr}}$, to terms such as (1) the binary transverse motion of the pulsar, $v_{\text {bin, } \perp}$, (2) the component of the Earth's

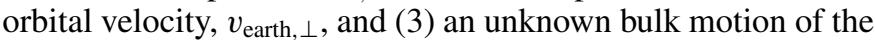
screen, $v_{\text {screen }}$. Including the pulsar binary motion, Equation (4) of Gupta (1995) can be rewritten as

$$
V_{\mathrm{iss}}=\left|x V_{\mu}+v_{\mathrm{bin}, \perp}+v_{\mathrm{earth}, \perp}-(1+x) v_{\text {screen }}\right| .
$$

For PSR J0437-4715, the error on $V_{\text {iss }}$ is largely due to measurement errors on $v_{\mathrm{d}}$ and $\tau_{\text {iss. }}$. Even though refractive modulations may alter these quantities, on the basis of a predicted (and observed) positive correlation of their variabilities, we expect the $V_{\text {iss }}$ measurements to be relatively stable. Since $v_{\text {bin }} \sim 13 \mathrm{~km} \mathrm{~s}^{-1}$, even after accounting for a maximal contribution from the Earth's motion $\left(30 \mathrm{~km} \mathrm{~s}^{-1}\right)$ and a nominal $\sim 15 \mathrm{~km} \mathrm{~s}^{-1}$ for $v_{\text {screen }}$ (Bondi et al. 1994), we estimate $V_{\text {iss }} \sim$ $325 \mathrm{~km} \mathrm{~s}^{-1}$, which is on the higher end of the range of values derived from past observations.

As seen from Table 1, almost all measured values of $V_{\text {iss }}$ are consistently larger than the pulsar's transverse velocity, $V_{\mu} \sim 100 \mathrm{~km} \mathrm{~s}^{-1}$. Based on the formalism and the underlying lever-arm argument in deriving Equation (2), this would strongly suggest a screen location that is closer to the pulsar. Specifically, our measurement yields $x=V_{\text {iss }} / V_{\mu} \sim 3$, implying a scattering screen located at $\sim 120$ pc from the observer. A similar inference was made by Gothoskar \& Gupta (2000) based on their observations at $327 \mathrm{MHz}$ (Table 1), suggesting $D_{\mathrm{os}} \sim 0.6 D_{\mathrm{psr}}$. Conservatively, $x \sim 1-3$ from the published data so far, which would translate to a screen location $D_{\mathrm{os}} \sim(0.5-0.8) D_{\mathrm{psr}}$, i.e., $\sim 80-120 \mathrm{pc}$. The formalism of Deshpande \& Ramachandran (1998), which considers the more realistic case of a discrete scatterer and a distributed component, predicts $D_{\mathrm{os}} \sim(0.65-0.95) D_{\mathrm{psr}}$, with an implied $\theta_{\mathrm{H}} / \theta_{\tau} \sim$ $0.2-0.6$, the ratio of the source size to the scattering angle. Interestingly, this inferred screen location compares well with the estimated size of the Local Bubble in this direction, which is $\sim 100-120$ pc (Bhat et al. 1998). The bubble extends out to $\sim 10-50$ pc in the Galactic plane and $\sim 100-200$ pc perpendicular to the plane (e.g., Snowden et al. 1990; Bhat et al. 1998; Cordes \& Lazio 2002).

This is, however, in stark disagreement with Smirnova et al. (2006), who suggest a location $\sim 10$ pc from the Sun. Their conclusion relies on a better agreement of their estimated scattering angle, $\theta_{\text {diff }}$, from the observed scintillation timescale of Gwinn et al. (2006) (and some reasonable assumptions), with the predictions based on the statistical dependence of $\theta_{\text {diff }}-D M$ (from Pynzar' \& Shishov 1997). The empirical relation between scattering and DM is, however, poorly constrained at low DMs, $\lesssim 10 \mathrm{pc} \mathrm{cm}^{-3}$ (see Bhat et al. 2004). Furthermore, their relative refractive angle $\left(\theta_{\text {ref }} \sim 2 \theta_{\text {diff }}\right)$ is inconsistent with that inferred from our observations. For a midway location of the screen, we estimate $\theta_{\text {diff }} \sim 0.7$ mas and $\theta_{\text {ref }} \sim 0.3$ mas, and hence $\theta_{\text {ref }} \sim \theta_{\text {diff }} / 2$. Furthermore, a $\sim 10$ pc screen location would mean $x \sim D_{\mathrm{os}} / D_{\mathrm{ps}} \sim 0.1$, implying very low values of $V_{\mathrm{iss}}$, which is not supported by any of the observations so far.

\subsection{Pulse Profile Evolution with Radio Frequency}

Figure 4 presents the integrated pulse profiles of PSR J0437-4715 at frequencies from 0.2 to $17 \mathrm{GHz}$, spanning almost two orders of magnitude in frequency. Data at $438 \mathrm{MHz}$ are from Navarro et al. (1997), while those at higher frequencies are from the Parkes observatory pulsar data archive (Hobbs 


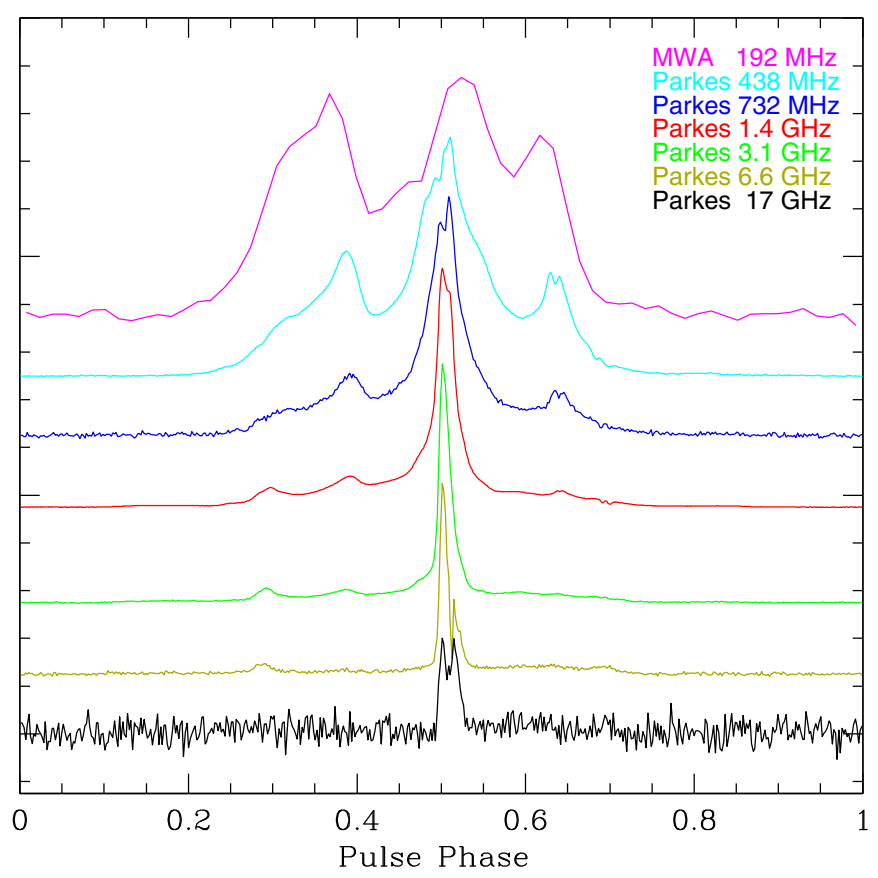

Figure 4. Integrated profiles of PSR J0437-4715 at frequencies from $200 \mathrm{MHz}$ to $17 \mathrm{GHz}$. Archival data from Parkes were processed to generate pulse profiles with 512 phase bins across the pulse period (i.e., a time resolution of $\approx 11.23 \mu \mathrm{s}$ ), whereas the MWA profile has 64 phase bins, i.e., approximately $90 \mu$ s time resolution.

(A color version of this figure is available in the online journal.)

et al. 2011). At frequencies from 0.4 to $3 \mathrm{GHz}$ where it has been intensely studied, the central bright (core) component is flanked by multiple outer (conal-like) components that are also asymmetric, with a characteristic "notch" clearly visible (in both total intensity and polarization) in the data taken at 0.43 and 1.3 GHz (Navarro et al. 1997; Dyks et al. 2007). Modeling work has identified at least seven clear components in its profile, i.e., three distinct cones surrounding a central core (Gil \& Krawczyk 1997; Qiao et al. 2002). The emission physics and beam modeling are difficult for pulsars in general, and with the observed complexity in polarization and the pulse profile, it becomes particularly challenging for PSR J0437-4715.

The pulse profiles were nominally aligned using a simple template matching technique (Taylor 1992) as implemented within PSRCHIVE. The peak of the core component is fairly well aligned in this process, except at $17 \mathrm{GHz}$, where the profile is significantly different and aligned near the center of the bridge emission.

The pulse shape is evidently complex, with the emission typically spanning $\pm 100^{\circ}$ in longitude around the core peak (Figure 4). The relative longitudinal phase shifts of the conal components are $\sim 0.05\left(20^{\circ}\right)$ between the leftward peaks (at $1.4 \mathrm{GHz}$ and $0.2 \mathrm{GHz})$ and $\sim 0.025\left(10^{\circ}\right)$ between the rightward peaks. These outer components evolve to become almost as strong as the core component at $0.2 \mathrm{GHz}$, indicating that their relative spectra are significantly different from those of the central components. Further, a mean flux of $\sim 1-2$ Jy indicated by our observations may imply a possible spectral break in the 200-400 MHz range (see McConnell et al. 1996).

The complex pulse shape of PSR J0437-4715 and its strong evolution with frequency make modeling in terms of emission beams challenging. Both the coherent curvature radiation (Gil \& Krawczyk 1997) and inverse Compton scattering (Qiao et al.
2002) models reproduce the observed pulse shapes (at 436 and $1382 \mathrm{MHz}$ ) with reasonable success, with an emission geometry comprising the core and multiple outer cones. However, the asymmetry seen with the outer conal components is not reproduced by either of the models. Furthermore, neither model takes into account the relative longitudinal phase shifts of the conal components, which Gangadhara \& Thomas (2006) interpret in terms of retardation-aberration effects and estimate the emission altitudes to be $\sim 10 \%-30 \%$ of the light cylinder radius.

The MWA detection also highlights the importance of accounting for spectral evolution of the pulsar emission in accurate determinations of DMs. At low frequencies, frequencydependent DM changes may also arise from multi-path propagation effects, as the radiation at different frequencies samples slightly different total electron contents $\left(\theta_{\text {diff }} \propto v_{\text {obs }}{ }^{-2.2}\right)$. Any unmodeled profile evolution may thus manifest as DM variations, as demonstrated by Hassall et al. (2012) and Ahuja et al. (2007), albeit for long-period pulsars. Recent work of Pennucci et al. (2014) involving a two-dimensional template portrait is very promising in this context.

DM variations $\sim 10^{-3} \mathrm{pc} \mathrm{cm}^{-3}$ have been seen toward PSR J0437-4715 over 5-10 yr, and even larger variations $\left(\sim 10^{-2} \mathrm{pc} \mathrm{cm}^{-3}\right)$ toward other pulsars (Keith et al. 2013). For typical DM accuracies achievable with the PPTA data $\left(\sim 10^{-4} \mathrm{pc} \mathrm{cm}^{-3}\right)$, the differential delay across the full MWA frequency range is $\approx 60 \mu \mathrm{s}$. The large frequency lever arm possible with the MWA, e.g., by spreading out $24 \times 1.28 \mathrm{MHz}$ channels of VCS non-contiguously over the $220 \mathrm{MHz}$ band, so as to simultaneously sample multiple spot frequencies within the 80-300 MHz range, can be exploited to enable such wide-band observations.

\section{FUTURE PROSPECTS}

With its sensitivity, field of view, and frequency coverage, the MWA is a major facility in low-frequency pulsar astronomy. Our current capabilities will soon be boosted with the implementation of the full-bandwidth recording and phased-array modes, resulting in over an order of magnitude improvement in sensitivity. The combination of the field of view and VCS functionality can be exploited for realizing observations of multiple timingarray pulsars from a given pointing, and the flexibility to spread out the $30.72 \mathrm{MHz}$ bandwidth anywhere within the $80-300 \mathrm{MHz}$ range can be leveraged for simultaneous observations at multiple frequencies.

We thank the referee for several insightful comments that helped improve the Letter. This scientific work makes use of the Murchison Radio-astronomy Observatory, operated by CSIRO. We acknowledge the Wajarri Yamatji people as the traditional owners of the Observatory site. N.D.R.B. is supported by a Curtin Research Fellowship. We thank Matthew Bailes and Bryan Gaensler for useful comments, and Dick Manchester and Lawrence Toomey for help with access to the $430 \mathrm{MHz}$ archival data. Support for the MWA comes from the U.S. National Science Foundation (grants AST-0457585, PHY-0835713, CAREER-0847753, and AST-0908884), the Australian Research Council (LIEF grants LE0775621 and LE0882938), the U.S. Air Force Office of Scientific Research (grant FA9550-0510247), and the Centre for All-sky Astrophysics (an ARC Centre of Excellence funded by grant CE110001020). Support is also provided by the Smithsonian Astrophysical Observatory, the MIT School of Science, the Raman Research Institute, the Australian National University, 
and the Victoria University of Wellington (via grant MEDE1799 from the New Zealand Ministry of Economic Development and an IBM Shared University Research Grant). The Australian Federal government provides additional support via CSIRO, National Collaborative Research Infrastructure Strategy, Education Investment Fund, and the Australia India Strategic Research Fund, and Astronomy Australia Limited, under contract to Curtin University. We acknowledge the iVEC Petabyte Data Store, the Initiative in Innovative Computing and the CUDA Center for Excellence sponsored by NVIDIA at Harvard University, and the International Centre for Radio Astronomy Research, a joint venture of Curtin University and The University of Western Australia, funded by the Western Australian State government.

\section{REFERENCES}

Abdo, A. A., Ajello, M., Allafort, A., et al. 2013, ApJS, 208, 17

Ahuja, A. L., Mitra, D., \& Gupta, Y. 2007, MNRAS, 377, 677

Bell, J. F., Bailes, M., \& Bessell, M. S. 1993, Natur, 364, 603

Bhat, N. D. R., Cordes, J. M., Camilo, F., Nice, D. J., \& Lorimer, D. R. 2004, ApJ, 605,759

Bhat, N. D. R., Gupta, Y., \& Rao, A. P. 1998, ApJ, 500, 262

Bhat, N. D. R., Rao, A. P., \& Gupta, Y. 1999, ApJS, 121, 483

Bondi, M., Padrielli, L., Gregorini, L., et al. 1994, A\&A, 287, 390

Cordes, J. M., \& Lazio, T. J. W. 2001, ApJ, 549, 997

Cordes, J. M., \& Lazio, T. J. W. 2002, arXiv:astro-ph/0207156

Cordes, J. M., \& Rickett, B. J. 1998, ApJ, 507, 846

Deller, A. T., Verbiest, J. P. W., Tingay, S. J., \& Bailes, M. 2008, ApJL, 685, L67

Deshpande, A. A., \& Ramachandran, R. 1998, MNRAS, 300, 577

Dyks, J., Rudak, B., \& Rankin, J. M. 2007, A\&A, 465, 981

Gangadhara, R. T., \& Thomas, R. M. C. 2006, arXiv:astro-ph/0604559

Gil, J., \& Krawczyk, A. 1997, MNRAS, 285, 561

Gothoskar, P., \& Gupta, Y. 2000, ApJ, 531, 345

Gupta, Y. 1995, ApJ, 451, 717

Gupta, Y., Bhat, N. D. R., \& Rao, A. P. 1999, ApJ, 520, 173
Gupta, Y., Rickett, B. J., \& Lyne, A. G. 1994, MNRAS, 269, 1035

Gwinn, C. R., Hirano, C., \& Boldyrev, S. 2006, A\&A, 453, 595

Hassall, T. E., Stappers, B. W., Hessels, J. W. T., et al. 2012, A\&A, 543, A66

Hobbs, G., Miller, D., Manchester, R. N., et al. 2011, PASA, 28, 202

Hotan, A. W., van Straten, W., \& Manchester, R. N. 2004, PASA, 21, 302

Issur, N. H., \& Deshpande, A. A. 2000, in ASP Conf. Proc. Vol. 202, IAU Colloq. 177: Pulsar Astronomy - 2000 and Beyond, ed. M. Kramer, N. Wex, \& N. Wielebinski (San Francisco, CA: ASP), 171

Johnston, S., Lorimer, D. R., Harrison, P. A., et al. 1993, Natur, 361, 613

Johnston, S., Nicastro, L., \& Koribalski, B. 1998, MNRAS, 297, 108

Kargaltsev, O., Pavlov, G. G., \& Romani, R. W. 2004, ApJ, 602, 327

Keith, M. J., Coles, W., Shannon, R. M., et al. 2013, MNRAS, 429, 2161

Lonsdale, C. J., Cappallo, R. J., Morales, M. F., et al. 2009, IEEEP, 97, 1497

Manchester, R. N., Hobbs, G., Bailes, M., et al. 2013, PASA, 30, 17

Manchester, R. N., \& Johnston, S. 1995, ApJL, 441, L65

McConnell, D., Ables, J. G., Bailes, M., \& Erickson, W. C. 1996, MNRAS, 280,331

Navarro, J., Manchester, R. N., Sandhu, J. S., Kulkarni, S. R., \& Bailes, M. 1997, ApJ, 486, 1019

Nicastro, L., \& Johnston, S. 1995, MNRAS, 273, 122

Pennucci, T. T., Demorest, P. B., \& Ransom, S. M. 2014, arXiv:1402.1672

Phillips, J. A., \& Clegg, A. W. 1992, Natur, 360, 137

Pynzar', A. V., \& Shishov, V. I. 1997, ARep, 41, 586

Qiao, G.-J., Wang, X.-D., Wang, H.-G., \& Xu, R.-X. 2002, ChJAA, 2, 361

Ransom, S. M. 2001, PhD Thesis, Harvard Univ.

Rickett, B. J., Lyne, A. G., \& Gupta, Y. 1997, MNRAS, 287, 739

Smirnova, T. V., Gwinn, C. R., \& Shishov, V. I. 2006, A\&A, 453, 601

Snowden, S. L., Cox, D. P., McCammon, D., \& Sanders, W. T. 1990, ApJ, 354,211

Spangler, S. R. 2009, SSRv, 143, 277

Taylor, J. H. 1992, RSPTA, 341, 117

Tingay, S. J., Goeke, R., Bowman, J. D., et al. 2013, PASA, 30, 7

van Straten, W. 2013, ApJS, 204, 13

van Straten, W., \& Bailes, M. 2011, PASA, 28, 1

Verbiest, J. P. W., Bailes, M., van Straten, W., et al. 2008, ApJ, 679, 675

Vivekanand, M., Ables, J. G., \& McConnell, D. 1998, ApJ, 501, 823

Wang, N., Manchester, R. N., Johnston, S., et al. 2005, MNRAS, 358, 270

Yan, W. M., Manchester, R. N., van Straten, W., et al. 2011, MNRAS, 414, 2087

Zavlin, V. E., Pavlov, G. G., Sanwal, D., et al. 2002, ApJ, 569, 894 